\title{
RATIONALLY VARYING POLARIZING SUBALGEBRAS IN NILPOTENT LIE ALGEBRAS
}

\author{
LAWRENCE CORWIN AND FREDERICK P. GREENLEAF ${ }^{1}$
}

\begin{abstract}
Let $\mathfrak{R}$ be a nilpotent Lie algebra, with (vector space) dual $\mathfrak{R}^{*}$. We construct a map $l \mapsto \mathfrak{M}$, from a Zariski-open subset of $\mathfrak{R}^{*}$ to the set of subalgebras of $\mathfrak{R}$ such that $\mathfrak{M}_{l}$ varies rationally with $l, \mathfrak{M}_{l}$ is polarizing for $l,(\operatorname{Ad} x) \mathfrak{M}_{l}=\mathfrak{M}_{l}$, $\left(l^{\prime}=\operatorname{Ad}^{*} x \cdot l\right)$ for all $x \in N$, and $\mathfrak{B}_{\infty}(l) \subseteq \mathfrak{M}, \subseteq \Re_{\infty}(l)$, where $\mathfrak{B}_{\infty}(l)$ and $\Omega_{\infty}(l)$ are the canonical subalgebras introduced by $R$. Penney.
\end{abstract}

1. Let $N$ be a connected, simply connected nilpotent Lie group with Lie algebra $\mathfrak{N}$; for $l \in \mathfrak{N}^{*}$, define the bilinear form $B_{l}$ by $B_{l}(X, Y)=l([X, Y])$. In the Kirillov theory of the unitary representations of $N$, an important role is played by a polarizing (or maximal subordinate) subalgebra of $l$-that is, a subalgebra $\mathfrak{M}$, which is a maximal isotropic subspace for $B_{l}$. As Kirillov [4] showed, such a subalgebra always exists; in general, it is not unique. For Kirillov's purposes, the lack of uniqueness was unimportant. He showed that if one defines $\chi_{l}$ on $M_{l}=\exp \mathfrak{M}$, by $\chi_{l}(\exp X)=\exp 2 \pi i l(X)$ and induces $\chi_{l}$ to a representation $\pi_{l}$ on $N$, then $\pi_{l}$ is independent (up to equivalence) of the choice of $\mathfrak{M}_{l}$; moreover, $\pi_{l}$ is irreducible, and every irreducible (unitary) representation of $N$ is equivalent to some $\pi_{l}$. Indeed, the unitary dual $\hat{N}$ of $N$ is homeomorphic to the orbit space of $\mathfrak{N}^{*} \bmod$ the coadjoint action of $N$; see [1].

For some purposes, it is useful to find concrete realizations of "most" of the irreducible representations $\pi_{l} \in N^{\wedge}$ which vary smoothly with $l$. Here, "most" means "for all $l$ in a Zariski-open set of $\mathfrak{N}^{*}$ "; see [6, p. 55ff] for a discussion. This problem has arisen in discussions of applications of representation theory to partial differential equations; it also arises when one attempts to characterize the Fourier transform of Schwartz class functions on nilpotent Lie groups. (See, e.g., [2] and [7]; in these cases, one was able to avoid serious problems because of the nature of the groups under discussion.)

In [8], [9] $\mathrm{M}$. Vergne showed that there is a natural construction which yields polarizing subalgebras $\mathfrak{M}_{l}$, which vary rationally in $l$. (Her construction actually works for solvable Lie algebras, where additional conditions are imposed on the $\mathfrak{M}_{\text {l.) }}$ ) For our purposes we seek polarizations which not only vary rationally in $l$, and covariantly under $\operatorname{Ad}^{*}(N)$, but which are also related to the canonical

Received by the editors November 27, 1978 and, in revised form, February 9, 1979.

1980 Mathematics Subject Classification. Primary 17B30, 22 E27.

Key words and phrases. Rationally varying polarizing subalgebras, nilpotent Lie algebras, canonical subalgebras.

${ }^{1}$ Research supported in part by NSF grants MCS 78-02715 and MCS 78-02153 respectively. 
subalgebras $\mathfrak{G}_{\infty}(l) \subseteq \Re_{\infty}(l)$ introduced by Penney [5], and used to advantage in [3]. We show that the canonical objects $\mathfrak{Q}_{\infty}(l) \subseteq \mathfrak{R}_{\infty}(l)$ all depend rationally on $l$, and that there is a rational choice of polarization that fits between them: $\mathfrak{B}_{\infty}(l) \subseteq \mathfrak{M}_{l}$ $\subseteq \Re_{\infty}(l)$ for all generic $l$. An example shows that the polarizations described in [8], [9] do not always satisfy this condition.

We would like to thank M. Duflo for pointing out the work in [8], [9].

2. We begin with some generalities. Let $E$ be a finite-dimensional real vector space, and let $U \subseteq E$ be Zariski-open. Suppose that $W$ is some other real finite-dimensional vector space. We say that the field of vectors $\varphi: U \rightarrow W$ is rational if the coefficients of $\varphi$ (with respect to any fixed bases of $E, W$ ) are rational functions of $v \in U$. A field of subspaces $\left\{W_{v}: v \in U\right\}$ is rational if there are rational fields $\varphi_{1}, \ldots, \varphi_{k}: U \rightarrow W$ which form a basis for $W_{v}$ on a Zariski-open subset of $U$. Note that a field of linear operators $T: E \rightarrow \operatorname{Hom}(V, W)$ may be regarded as a field of vectors.

In what follows, $E$ will always be the indexing vector space. We shall often suppress mention of the Zariski-open set $U$; in fact, $U$ may vary somewhat during the discussion. We use "generic" to mean "Zariski-open". We shall use, e.g., $W_{v}$ and $W(v)$ interchangeably to refer to the value at $v$ of the field $\left\{W_{v}\right\}$ of subspaces.

2.1. Lemma. Let $\varphi_{1}, \ldots, \varphi_{j}$ be rational vector fields on $W$ which are linearly independent at a point $v_{0} \in E$. Then they are generically linearly independent, and there are rational vector fields $\varphi_{j+1}, \ldots, \varphi_{n}$ such that $\left\{\varphi_{1}(v), \ldots, \varphi_{n}(v)\right\}$ is a basis of $W$ for generic $v \in E$.

Proof. Extend $\varphi_{1}\left(v_{0}\right), \ldots, \varphi_{j}\left(v_{0}\right)$ to a basis $\varphi_{1}\left(v_{0}\right), \ldots, \varphi_{j}\left(v_{0}\right), w_{j+1}, \ldots, w_{n}$, and define $\varphi_{i}(v)=w_{i}$ for $j+1 \leqslant i \leqslant n$. It suffices to show that $\left\{\varphi_{1}(v), \ldots, \varphi_{n}(v)\right\}$ is a basis for generic $v$, and this is clear from the fact that $\operatorname{Det}\left(\varphi_{1}, \ldots, \varphi_{n}\right)$ is rational and not identically 0 .

2.2. LeMma. Let $\varphi_{1}, \ldots, \varphi_{n}$ be rational vector fields on $W$ which form a basis for generic $v$. Define vector fields $\psi_{1}, \ldots, \psi_{n}$ on $W^{*}$ such that for generic $v$, $\psi_{1}(v), \ldots, \psi_{n}(v)$ is the dual basis to $\varphi_{1}(v), \ldots, \varphi_{n}(v)$. Then $\psi_{1}, \ldots, \psi_{n}$ are rational.

Proof. This is a simple application of Cramer's rule.

2.3. Lemma. Let $\left\{W_{v}\right\}$ be a rational field of subspaces in $W$. Then $\left\{W_{v}^{\perp}\right\}$ is a rational field of subspaces in $W^{*}$.

Proof. Let $\varphi_{1}, \ldots, \varphi_{k}$ be the rational fields which give a basis for the $W_{v}$ at generic $v$. Extend these to a generic basis $\left\{\varphi_{1}, \ldots, \varphi_{n}\right\}$ of $W$, as in Lemma 2.1, and let $\left\{\psi_{1}, \ldots, \psi_{n}\right\}$ be the dual basis of fields in $W^{*}$, rational by Lemma 2.2. Then $W_{v}^{\perp}=\operatorname{span}\left(\psi_{k+1}(v), \ldots, \psi_{n}(v)\right)$ for generic $v$, and the lemma is proved.

2.4. Proposition. Let $T: E \rightarrow \operatorname{Hom}\left(W_{1}, W_{2}\right)$ be a rational field of linear operators. Then

(a) $T^{*}: E \rightarrow \operatorname{Hom}\left(W_{2}^{*}, W_{1}^{*}\right)$, where $T^{*}(v)=(T(v))^{*}$, is rational;

(b) Range $T$ is a rational field of subspaces in $W_{2}$; 
(c) Ker $T$ is a rational field of subspaces in $W_{1}$;

(d) Rank $T$ takes on its maximum value generically;

(e) $\operatorname{Null}(T)$ takes on its minimum value generically.

Proof. For (a), choose bases in $W_{1}$ and $W_{2}$, and use the dual bases in $W_{2}$ and $W_{1}$; as the matrix for $T^{*}$ is the transpose of the matrix for $T$, the result follows. Next, (c) and (e) follow from (b) and (d), plus the familiar formula $\operatorname{Ker} T=$ (Range $\left.T^{*}\right)^{\perp}$ and Lemma 2.3 .

So it suffices to prove (b) and (d). For (b), choose $v_{0}$ such that $T\left(v_{0}\right)$ has maximal rank; choose vectors $w_{1}, \ldots, w_{k} \in W_{1}$ such that $\left\{T\left(v_{0}\right) w_{j}\right\}$ is a basis for Range $T\left(v_{0}\right)$. Then $U=\left\{v=\left\{T(v) w_{j}: 1 \leqslant j \leqslant k\right\}\right.$ is linearly independent $\}$ is Zariski-open, and for $v \in U$, the fields $\varphi_{j}(v)=T(v) w_{j}$ span Range $T(v)$ (since $\operatorname{Rank} T\left(v_{0}\right)$ is maximal). Thus Range $T$ is rational. For (d), note that the set where Rank $T$ is maximal is a union of sets like $U$.

3. In dealing with fields of subalgebras, we shall consider two situations: first, a fixed nilpotent Lie algebra $\mathfrak{N}$, along with a rational field $\left\{\mathfrak{R}_{v}\right\}$ of subalgebras; and second, a fixed space $V=\mathbf{R}^{k}$, along with Lie algebra structures depending rationally on a parameter $v \in E$. (That is, the structure constants with respect to a fixed basis are rational functions of $v$.) It is convenient to subsume the first case under the second. Let $\varphi_{1}, \ldots, \varphi_{k}$ be rational vector fields which (generically) give a basis for the $\mathfrak{N}_{v}$; for generic $v$,

$$
\left[\varphi_{i}(v), \varphi_{j}(v)\right]=\sum_{h=1}^{k} c_{h i j}(v) \varphi_{h}(v), \quad 1 \leqslant h, i, j<k,
$$

where the $c_{h i j}$ are rational functions of $v$. Now fix a basis $e_{1}, \ldots, e_{k}$ of $\mathbf{R}^{k}$, and define

$$
\left[e_{i}, e_{j}\right]_{v}=\sum_{h=1}^{k} c_{h i j}(v) e_{h}
$$

we obtain a rational field of Lie algebra structures on $\mathbf{R}^{k}$ isomorphic (in an obvious sense) to the field $\left\{\mathfrak{R}_{v}\right\}$.

If $\mathfrak{R}$ is a Lie algebra and $\mathfrak{\Im}$ is an ideal, we define $3_{\mathfrak{J}}(\mathfrak{N})=\{X \in \mathfrak{N}:[X, \mathfrak{N}] \subseteq$ $\mathfrak{\Im}$; ; in particular, $\mathfrak{Z}_{(0)}(\mathfrak{N})=\mathfrak{3}(\mathfrak{N})$, the center of $\mathfrak{N}$. If $l \in \mathfrak{N}^{*}$, then we define

$$
\begin{aligned}
& \mathfrak{R}_{l}=\text { radical of } B_{l}=\{X \in \mathfrak{R}: l([X, \mathfrak{R}])=0\} \text {, } \\
& \mathfrak{S}_{l}(\mathfrak{R})=\mathfrak{S}_{l}=\mathfrak{B}_{l}^{(1)}=\text { ideal generated by } \mathfrak{R}_{l} \text {, } \\
& \mathfrak{S}_{l}^{(j+1)}=\mathfrak{S}_{l \mid \oiint^{(j)}}\left(\mathfrak{Q}_{l}^{(j)}\right) \text {. }
\end{aligned}
$$

(See [5] for a discussion of the $\mathfrak{S}^{(j)}$.) We shall also write $\mathfrak{R}(l)$, etc., for typographical convenience.

3.1. TheOREm. Let $\mathfrak{R}$ be a nilpotent Lie algebra, $\left\{\mathfrak{N}_{v}: v \in E\right\}$ a rational field of subalgebras, $\left\{\mathfrak{\Im}_{v}: v \in E\right\}$ a rational field of subalgebras such that $\mathfrak{\Im}_{v}$ is an ideal of $\mathfrak{N}_{v}$ for generic $v$, and $\left\{l_{v}: v \in E\right\}$ a rational field of elements of $\mathfrak{N}^{*}$. We regard $l_{v}$ as restricted to $\mathfrak{N}_{v}$. Then the following fields are rational: 

(a) $3_{\Im_{0}}\left(\mathfrak{R}_{v}\right)$;
(b) $\Re_{l}\left(\subseteq \Re_{v}\right)$;
(c) $\mathfrak{S}_{l_{0}}^{(j)}, j=1,2, \ldots$

Proof. Let $k$ be the generic dimension of $\mathfrak{N}_{\alpha}$; let $\left\{e_{1}, \ldots, e_{k}\right\}$ be the standard basis of $\mathbf{R}^{k}$. As noted previously, we may regard the $\Re_{v}$ as modeled on $\mathbf{R}^{k}$; we may also assume that (generically) $\mathfrak{\Im}_{v}=\operatorname{span}\left(e_{1}, \ldots, e_{s}\right)$.

(a) Let $\left[X, e_{i}\right]_{v}=\sum_{j=1}^{k} c_{i j}(v ; X) e_{j}, X \in \mathbf{R}^{k}$. Then $\mathfrak{Z}_{\mathfrak{Y}_{v}}\left(\mathfrak{N}_{v}\right)$ is the kernel of the map $T_{v}: \mathbf{R}^{k} \rightarrow \mathbf{R}^{k(k-s)}$ defined by

$$
T_{v}(X)=\left(c_{i j}(v ; X): 1<i<k, s<j<k\right) .
$$

(b) $\Re_{l_{0}}$ is the kernel of $S_{v}: \mathbf{R}^{k} \rightarrow \mathbf{R}^{k}$, defined by

$$
S(X)=\left(l_{v}\left(\left[e_{1}, X\right]_{v}\right), \ldots, l_{v}\left(\left[e_{k}, X\right]_{v}\right)\right)
$$

In both (a) and (b), we may now apply Proposition 2.4.

(c) It suffices to prove the result for the case $j=1$, and then to apply induction. We may assume that $s=\operatorname{dim} \Re\left(l_{v}\right)$ is constant. Let $\varphi_{1}, \ldots, \varphi_{s}$ be rational vector fields giving bases for generic $v$. Choose $v_{0}$ with $\operatorname{dim} \oiint_{\mathcal{B}}\left(l_{v_{0}}\right)$ maximal, and choose indices $i_{1}, \ldots, i_{p}, j_{1}, \ldots, j_{p}(1 \leqslant p \leqslant r)$, such that $\left\{\varphi_{1}\left(v_{0}\right), \ldots, \varphi_{s}\left(v_{0}\right)\right.$, $\left.\left[e_{i_{1}}, \varphi_{j_{1}}\left(v_{0}\right)\right], \ldots,\left[e_{i_{r}}, \varphi_{j_{r}}\left(v_{0}\right)\right]\right\}$ is a basis for $\mathscr{B}\left(l_{v_{0}}\right)$. Then the $\varphi_{j}$ and $\psi_{p}$ (where $\left.\psi_{p}(v)=\left[e_{i_{p}}, \varphi_{j_{p}}(v)\right]\right)$ are rational vector fields giving a basis for $\$\left(l_{v}\right)$ for generic $v$.

Recall (from [5]; see also [3]) that for fixed $v$, the $\mathfrak{S}_{l_{0}}^{(j)}$ are constant for large $j$ (for $j \geqslant \operatorname{dim} n$, say); we denote this fixed algebra by $\mathscr{G}^{\infty}\left(l_{v}\right)$. Its annihilator for the form $B_{l_{0}}$ is denoted by $\AA^{\infty}\left(l_{v}\right) ; \mathfrak{G}^{\infty}\left(l_{v}\right)$ and $\AA^{\infty}\left(l_{v}\right)$ are both subalgebras and $\mathfrak{G}^{\infty}\left(l_{v}\right)$ is the radical of $l_{v} \mid \AA^{\infty}\left(l_{v}\right)$. Furthermore, $\mathscr{S}^{\infty}\left(l_{v}\right)$ is an ideal in $\AA^{\infty}\left(l_{v}\right)$, and any subalgebra of $l_{v} \mid \AA^{\infty}\left(l_{v}\right)$ also polarizes $l_{v}\left(\right.$ on $\left.\Re_{v}\right)$.

3.2. Corollary. $\mathfrak{Q}^{\infty}\left(l_{v}\right)$ and $\Re^{\infty}\left(l_{v}\right)$ depend rationally on $v$.

Proof. The first half is the case $j=n$ of Theorem 3.1(c). For the second, let $\left\{\varphi_{j}=1 \leqslant j \leqslant r\right\}$ be a field of vectors which generically gives a basis for $\oiint_{\mathcal{G}^{\infty}}\left(l_{v}\right)$. Then $\Re^{\infty}\left(l_{v}\right)=\operatorname{ker} T_{v}$, where

$$
T_{v}(X)=\left(l\left(\left[\varphi_{1}(v), X\right]\right), \ldots, l\left(\left[\varphi_{r}(v), X\right]\right)\right) .
$$

4. The main results of this paper are consequences of the following lemma.

4.1. Lemma. Let $\left\{\mathfrak{R}_{v}\right\},\left\{\mathfrak{\Im}_{v}\right\}$ be rational fields of subalgebras of a nilpotent Lie algebra $\mathfrak{N}$ such that $\mathfrak{J}_{v}$ is (generically) an ideal of $\mathfrak{N}_{v}$; let $\left\{l_{v}\right\}$ be a rational field of elements of $\mathfrak{R}^{*}$ such that $\mathfrak{\Im}_{v}$ is (generically) the radical of $B_{l_{v}} \mid \mathfrak{N}_{v} \times \mathfrak{N}_{v}$. Then there is a rational field $\left\{\mathfrak{M}_{v}\right\}$ of subalgebras such that (generically) $\mathfrak{M}_{v}$ polarizes $l_{v} \mid \mathfrak{R}_{v}$.

Proof. We may assume that the $\mathfrak{R}_{v}$ all have the same dimension, as do the $\mathfrak{\Im}_{v}$; let $\operatorname{dim}\left(\mathfrak{R}_{v}\right)-\operatorname{dim} \mathfrak{\Im}_{v}=2 m$. The proof is by induction on $m$, the case $m=0$ being trivial. If $m>0$, we use Theorem 3.1(a) to pick a rational vector field $\varphi$ such that

$$
\varphi\left(l_{v}\right) \in 3_{\mathfrak{\Im}\left(l_{0}\right)}\left(\mathfrak{N}\left(l_{v}\right)\right), \quad \varphi\left(l_{v}\right) \notin \Im\left(l_{v}\right) .
$$


Let $\mathfrak{\Im}_{1}\left(l_{v}\right)=\operatorname{span}\left(\Im\left(l_{v}\right), \varphi\left(l_{v}\right)\right)$, and let $\mathfrak{R}_{1}\left(l_{v}\right)=\mathfrak{\Im}_{1}\left(l_{v}\right)^{\perp}$ (relative to $B_{b}$ on $\mathfrak{R}_{v}$ ). Then $\mathfrak{J}_{1}\left(l_{v}\right) \triangleleft \mathfrak{N}\left(l_{v}\right)$, and $a$ fortiori $\mathfrak{J}_{1}\left(l_{v}\right) \triangleleft \mathfrak{N}_{1}\left(l_{v}\right)$; also, $\mathfrak{\Im}_{1}\left(l_{v}\right)$ is the radical of $B_{l_{0}} \mid \mathfrak{R}_{1}\left(l_{v}\right)$ $\times \mathfrak{R}_{1}\left(l_{v}\right)$, as a dimension-counting argument shows. Clearly $\mathfrak{\Im}_{1}\left(l_{v}\right)$ is rational, and $\mathfrak{R}_{1}\left(l_{v}\right)$ is rational by an argument like that used in Corollary 2.2. As $\operatorname{dim} \mathfrak{R}_{1}\left(l_{v}\right)-$ $\operatorname{dim} \mathfrak{\Im}_{1}\left(l_{v}\right)=2(m-1)$, the induction is complete.

4.2. TheOREM. Let $n$ be a nilpotent Lie group. Then there is a rational field $\left\{\mathfrak{M}_{l}: l \in \mathfrak{N}^{*}\right\}$ of subalgebras such that for all $l$ in a certain Zariski-open set $U \subseteq \mathfrak{N}^{*}, \mathfrak{M}_{l}$ is polarizing for $l$ and $\mathfrak{B}_{\infty}(l) \subseteq \mathfrak{M}_{l} \subseteq \mathfrak{R}_{\infty}(l)$.

Proof. We let $E=\mathfrak{R}^{*}$ in Corollary 3.2 and find that $\mathfrak{S}^{\infty}(l), \Re^{\infty}(l)$ depend rationally on $l$. Now the theorem is an immediate consequence of Lemma 4.1.

The Zariski-open set $U$ in Theorem 4.2 is not $\operatorname{Ad}^{*}(N)$-invariant, and the $\mathfrak{M}$, are not covariant with repect to Ad. We may remedy these defects at the cost of a bit more work. It is implicit in the discussion from [6] cited previously (some further details are spelled out in [2]) that if the dimension of a generic $\operatorname{Ad}^{*}(N)$-orbit in $\mathfrak{N}$ is $\mathfrak{\Im}$, then there is a subspace $W$ of codimension $\mathfrak{\Im}$ in $\mathfrak{N}$ and a birational map $F$ : $W \times \mathbf{R}^{d} \rightarrow \mathfrak{R}$ such that

(1) $W \cap U=U_{0}$ is a nonvoid Zariski-open subset of $W$;

(2) for $l \in U_{0}, F\left(l \times \mathbf{R}^{d}\right)=\operatorname{Ad}^{*}(N) l$. Indeed, there is a birational map $G$ : $W \times \mathbf{R}^{d} \rightarrow N$ such that for $l \in U_{0}, F(l, t)=\operatorname{Ad}^{*}(G(l, t)) l$. Find rational $\mathfrak{M}_{l_{0}}$ for $l_{0} \in U_{0}$ as in Theorem 4.2; for $l=F\left(l_{0}, t\right)$, define $\mathfrak{M}_{l}=\operatorname{Ad}(G(l, t)) \mathfrak{M}_{l_{0}}$. Set $U_{1}=$ $F\left(U_{0} \times \mathbf{R}^{d}\right)$. We have thus proved

4.3. TheOREM. Let $\mathfrak{R}$ be a nilpotent Lie group. Then there are a Zariski-open subset $U_{1} \subseteq \mathfrak{N}^{*}$ and a rational field $\left\{\mathfrak{M}_{l}: l \in \mathfrak{N}^{*}\right\}$ of subalgebras such that for all $l \in U_{1}$,

(a) $\mathfrak{M}_{l}$ is polarizing for $l$, and $\mathfrak{B}_{\infty}(l) \subseteq \mathfrak{M}_{l} \subseteq \mathfrak{R}_{\infty}(l)$,

(b) if $l^{\prime}=\left(\operatorname{Ad}^{*} n\right) l, n \in N$, then $l^{\prime} \in U_{1}$, and $\mathfrak{M}_{l^{\prime}}=(\operatorname{Ad} n) \mathfrak{M}_{l}$.

This theorem, and indeed all the results of this paper, hold equally for nilpotent algebraic groups over any field of characteristic 0 .

4.4. EXAMPLE Let $\mathfrak{N}$ be the $4 \times 4$ upper triangular matrices with zeros on the diagonal, let $E_{i j}$ have a 1 in the $(i, j)$ place and zeros elsewhere, and define ideals

$$
\begin{gathered}
\mathfrak{A}_{0}=(0), \quad \mathfrak{A}_{1}=\mathbf{R} E_{14}, \quad \mathfrak{A}_{2}=\mathfrak{A}_{1}+\mathbf{R} E_{13}, \quad \mathfrak{A}_{3}=\mathfrak{A}_{2}+\mathbf{R} E_{12}, \\
\mathfrak{A}_{4}=\mathfrak{A}_{3}+\mathbf{R} E_{24}, \quad \mathfrak{A}_{5}=\mathfrak{A}_{4}+\mathbf{R} E_{23} .
\end{gathered}
$$

Then the $\mathfrak{A}_{1}, \mathfrak{A}_{2}, \mathfrak{A}_{3}$ are abelian ideals and will lie in the $\mathfrak{M}_{l}$, described in [8]; for generic $l, \mathfrak{Q}_{\infty}(l)=\mathfrak{\Re}_{\infty}(l)=\mathbf{R} E_{13}+\mathbf{R} E_{14}+\mathbf{R} E_{23}+\mathbf{R} E_{24}$.

\section{BIBLIOGRAPHY}

1. Ian Brown, Dual topology of a nilpotent Lie group, Ann. Sci. École Norm. Sup. (4 Série) 6 (1973), 407-411.

2. L. Corwin and F. P. Greenleaf, Fourier transforms of smooth functions on certain nilpotent groups, J. Funct. Anal. 37 (1980), 203-217.

3. L. Corwin, F. P. Greenleaf and R. Penney, A canonical formula for the distribution kernels of primary projections in $L^{2}$ of a nilmanifold, Comm. Pure Appl. Math. 30 (1977), 355-372.

4. A. A. Kirillov, Unitary representations of nilpotent Lie groups, Uspehi Mat. Nauk 17 (1962), 57-110. 
5. R. Penney, Canonical objects in the Kirillow theory of nilpotent Lie groups, Proc. Amer. Math. Soc. 66 (1977), 175-178.

6. L. Pukanszky, Lecons sur les représentations des groups, Dunod, Paris, 1967.

7. C. Rockland, Hypoellipticity on the Heisenberg group-representation-theoretic criteria, Trans. Amer. Math. Soc. 240 (1978), 1-52.

8. M. Vergne, Construction de sous-algèbres subordonnées à un élément du dual d'une algèbre de Lie résoluble, C. R. Acad. Sci. Paris Sér. A-B 270 (1970), A173-A175.

9. Construction de sous-algèbres subordonnées à un élément du dual d'une algèbre de Lie résoluble, C. R. Acad. Sci. Paris Sér. A-B 270 (1970), A704-A707.

Department of Mathematics, Rutgers University, New Brunswick, New Jersey 08903

Courant Institute of Mathematical Sciences, New York University, New York, New York 10012 